\title{
An Estimation Method of the Number of On-board Passengers Applicable to Evaluation of Traffic Rescheduling Plans
}

\author{
Chikara HIRAI \\ Researcher, \\ Norio TOMII \\ Chief Researcher, \\ Planning Systems G., Transport Information Technology Div.
}

\begin{abstract}
We propose a method to estimate the number of on-board passengers for a given train traffic rescheduling plan. Based on the estimates by this method, we can calculate the evaluation indices of the rescheduling plan from the viewpoint of passengers. Our method is composed of two interactive simulations. One is a train traffic simulation that represents the movements of trains based on the given rescheduling plan. The other is a passengers flow simulation that represents passengers' choices for their own desirable trains based on the Logit model. We show how our method works with a simple example that clarifies inherent problems.
\end{abstract}

Keywords : simulation, estimation, passengers flow, Logit model, evaluation, train traffic rescheduling

\section{Introduction}

In most urban railway lines in Japan, a number of trains are operated at intervals of three to four minutes. When train traffic is disrupted by an accident, for example, rescheduling operations are conducted to aim at restoring normal train operation. This task is called the train traffic rescheduling (traffic rescheduling, in short).

On railway lines, especially on lines with heavy train traffic, a poor rescheduling plan imposes great inconvenience to a huge number of passengers. Thus, it is quite important to provide rescheduling plans of good quality.

Experts called "train dispatchers" reschedule disrupted traffic. They forecast the future train traffic based on the original train schedule and the current situation, and prepare a traffic rescheduling plan by making a series of appropriate changes to the original schedule.

One of the problems in the current process of traffic rescheduling is that no quantitative evaluation methods have been developed for traffic rescheduling plans and traffic rescheduling plans are put into practice with no attention paid to their quality. Train dispatchers do their jobs based on their past experience and intuition. They do not employ any method to select the best plan after making a quantitative comparison between several rescheduling plans. Hence, no one knows whether the adopted rescheduling plan is the best.

We believe that a quantitative evaluation method has to be established in order to make the traffic rescheduling work more reasonable. It is extremely important that the viewpoint of passengers' inconvenience is included in the criteria.

It is considered to be natural to adopt the total traveling time and the congestion of trains as the criteria of traffic rescheduling from the viewpoint of passengers. This implies the necessity of a method to estimate the number of passengers on each train for a given traffic rescheduling plan.

As for the methods to estimate the number of passengers, the results of researches on the notion of User Equi- librium Assignment model are reported 1) 2). These researches are based on the following conditions.

(1) Trains exactly run by following the departure / arrival times prescribed in the original schedule.

(2) Passengers have the information concerning the congestion of all trains and decide their behavior (choice of trains) so that the value of their utility becomes a maximum.

(3) When train traffic is disrupted, passengers at a station choose the train that departs first from the station.

The first condition, however, does not hold in the situation where traffic has been rescheduled. When train traffic is disrupted, the number of passengers who en/ detrain sometimes becomes larger than in the normal state, and the increase of passengers augments the dwell times of trains longer than those prescribed in the original schedule. It is not appropriate to think that train schedules are fixed. It is also necessary to take into account that they are influenced by the number of passengers who en/detrain. Then, we have to consider a dynamic traffic environment that is the interaction between the flow of passengers and delay of trains.

The second condition does not hold either. Although it is reasonable to assume that the second condition holds in the case of commuter transport where passengers iterate the same behavior everyday, it does not hold in the situation where train traffic is rescheduled. This is because traffic rescheduling plans differ from time to time and passengers cannot get enough information about the traveling time and the congestion of trains.

The third condition is not suitable for representing the behavior of passengers' various choices of trains. Not all passengers want to get on the train which departs first after their appearance at the station, even when train traffic is disrupted. It is natural to consider that passengers' behavior depends on the current circumstances.

We propose a method to estimate the number of passengers on each train for a given traffic rescheduling plan. In order to construct this method, we have to ad- 


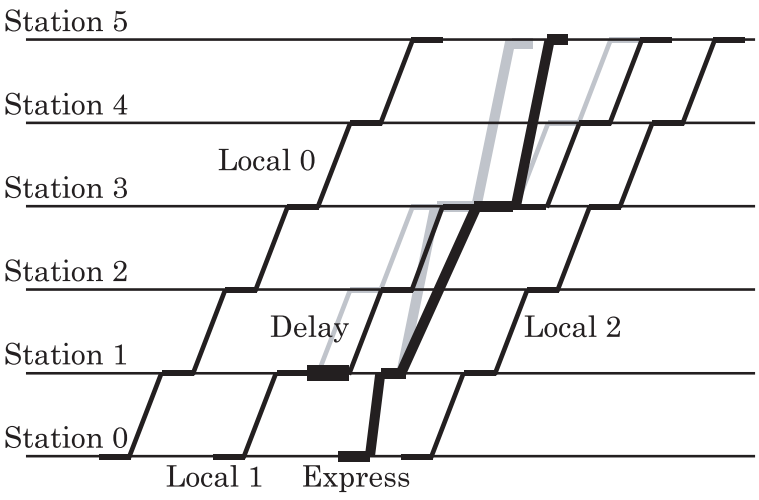

(a) Without any changes

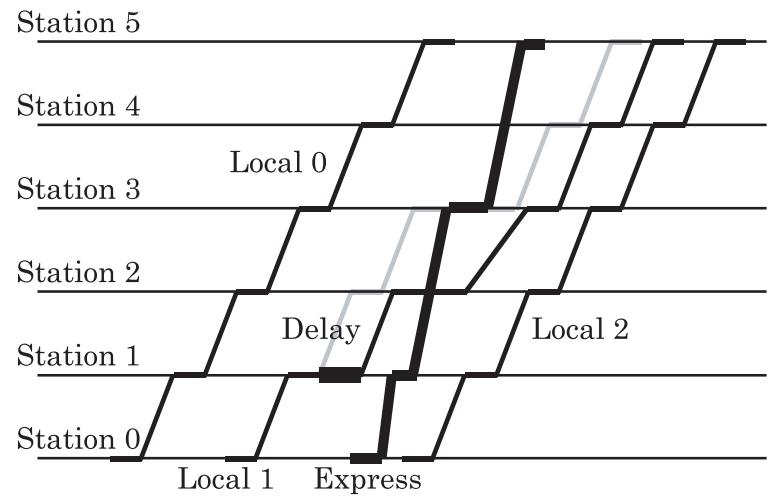

(b) Local 1 waits at Station 2

Fig. 1 Examples of rescheduling plans

dress the following issues.

(1) Representation of passengers' behavior including the transfer between trains.

(2) Representation of a dynamic traffic environment, i.e. the interaction between the flow of passengers and the delay of trains.

In this paper, we introduce a train traffic simulation model to solve the first issue. This can represent the interaction between the flow of passengers and the delay of trains. To settle the second issue, we devise a passenger flow simulation model based on the Logit model used commonly in the area of demand forecasting. We have implemented our simulation models, which makes it possible to estimate the number of passengers on each train under the dynamic traffic environment that considers the behavior of passengers.

\section{Evaluation of traffic rescheduling plans}

Traffic rescheduling plans should be evaluated from various viewpoints. Major criteria are the total delay time, the time needed for restoration of normal train operation and the number of cancelled trains. Among others, passengers' convenience should be the top priority. As the criteria to consider passengers' convenience, it is natural to adopt the total traveling time of passengers and the congestion of trains.

Figure 1 illustrates how traffic rescheduling plans should be evaluated from passengers' viewpoint. Gray lines denote the original schedule. Let us assume that a train named Local 1 is delayed for some reason at Station 1 . In this situation, there are two alternative rescheduling plans. One is to give no change to the original schedule, and leave the traffic to take its own course as shown in Fig. 1 (a). In this plan, a train named Express drawn by thick line will be delayed because Express has to pass Station 2 after the delayed departure of Local 1 at Station 2. The other is to change the schedule so that Express can pass Station 2 before Local 1 departs from Station 2 as in Fig. 1 (b). We call the former plan "Plan 1" and the latter "Plan 2."

The aim of Plan 1 is to keep the connection between
Local 1 and Express at Station 3. It is possible to reduce the traveling time of passengers who get on Local 1 bound for Station 5 at Station 2, because the passengers can transfer to Express at Station 3.

There are two aims of Plan 2. One is to reduce the traveling time of the passengers from Stations 0,1 and 3 who are bound for Station 5. The other is to avoid Express being delayed. If Express connected to Local 1 at Station 3, Express would be more crowded than in the normal state. The congestion of Express would cause augmentation of the dwell time of Express at Station 3 to delay the departure of Express.

We can discuss which plan is quantitatively better from the viewpoint of passengers, if we have the number of passengers on each train. In order to estimate the number of on-board passengers, we have to address the following two problems.

(1) How to represent passengers' behavior such as which train passengers choose.

(2) How to predict the future movement of trains as a result of the dynamic interaction between the flow of passengers and the delay of trains such as the fact that the increase of passengers augments the dwell time of trains.

For the first problem, we propose a model of passengers' behavior. The model is based on the Logit model that is often used in demand forecasting ${ }^{3)}$. The Logit model can be applied to represent the passengers' behavior such as the fact that some passengers choose a faster train and other passengers choose a less crowded train. The section 3.1 describes the detail of our passengers' behavior model.

For the second problem, we introduce a model that represents the interaction between delay of trains and congestion. This model is based on an experiment performed at the Railway Technical Research Institute ${ }^{4)}$.

Based on these two models, we have developed a simulator that can trace both the behavior of passengers and the movement of trains. It is a simulator that can estimate the number of on-board passengers. In the section 3.2 , the structure of the simulator is presented together with the detail of the answer to the second problem. 


\section{Estimation method of the number of on-board passengers}

\subsection{Passengers' behavior model}

3.1.1 Outline of the passengers' behavior model

We introduce the passengers' behavior model described in Fig. 2. The model consists of the following five steps, because the behavior of a railway passenger is considered to include the following steps.

(1) To appear (arrive) at a station and wait for a train.

(2) To decide whether to entrain.

(3) To entrain.

(4) To decide whether to detrain.

(5) To detrain.

A passenger's behavior between his/her appearance at the original station and his/her arrival at the destination station is represented by a combination of these five steps. For example, a passenger's transfer to another train is represented by a combination of "To decide whether to detrain," "To detrain," "To decide whether to entrain" and "To entrain."

\subsubsection{Application of the Logit model to the passengers} behavior model

We adopt the Logit model to predict passengers' choice of en/detraining. The Logit model is often used in the field of demand forecasting ${ }^{3)}$. This model gives the probability of a passenger's choice of a set of available routes and is based on an assumption that a passenger chooses the most desirable route for him/her. The formulation of the Logit model allows that a choice of routes is regarded as a passenger's choice of trains. In other words, it is possible to represent passengers' behavior by the Logit model.

Let $P(R)$ be the probability that a passenger choose a route $R$. If a passenger has two available routes $A$ and $B$,

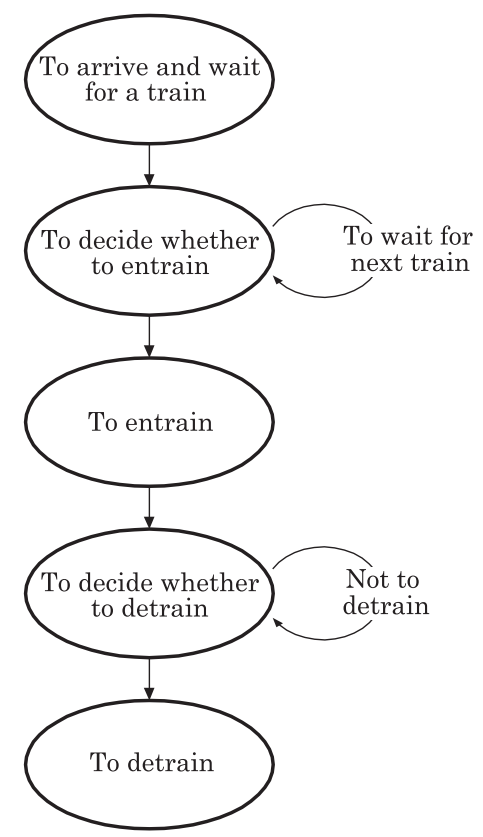

Fig. 2 Flow diagram of a passengers' behavior
$P(A)$ and $P(B)$ are denoted as follows ${ }^{5)}$.

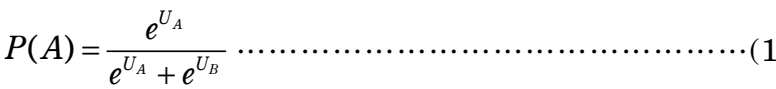

$$
\begin{aligned}
& P(B)=\frac{e^{U_{B}}}{e^{U_{A}}+e^{U_{B}}}
\end{aligned}
$$

where $e$ is the base of natural logarithm. $U_{R}$ means how desirable the route $R$ is for the passenger. That is, $U_{R}$ is the value of utilities of the passenger when the passenger chooses the route $R$. We assume that the value consists of the traveling time, the time needed for transfer to another train and the current congestion. Hence, the value of utilities is expressed by the following formula.

$U_{R}=a$ (Pseudo traveling time)

$+b$ (Necessary time to change of trains) $\cdots(3)$

where $a$ and $b$ are parameters. Congestion is included in "Pseudo traveling time" by using the following formula which expresses congestion by a measure of traveling time ${ }^{6)}$.

(Rate of additive traveling time)

$$
=\alpha(\text { Congestion rate })^{\beta .} .
$$

where $\alpha$ and $\beta$ are parameters. Then, we have the Pseudo traveling time as below.

$($ Pseudo traveling time $)=($ Traveling time $)$

$$
\left(1+\alpha(\text { Congestion rate })^{\beta}\right) \text {. }
$$

\subsection{Structure of the simulator}

Our estimation method for the number of on-board passengers is based on simulation techniques. We believe that simulation is the most appropriate way to represent the dynamic interaction between train traffic and passengers. Actually, our simulator consists of two simulators. One is a train traffic simulator (TTS) and the other is a passengers flow simulator (PFS). They share a memory where the train schedules are stored and these two simulators work hand in hand while exchanging messages and simulate the dynamic traffic environment formed by trains and passengers ${ }^{7}$. The configuration of the simulator is depicted in Fig. 3.

TTS simulates the movement of trains based on the given train schedule. It is designed based on the Colored Petri-net model ${ }^{8)}{ }^{9}$ ). TTS has several characteristics to always produce physically feasible simulation results. Automatic traffic rescheduling algorithms can easily be installed in it.

PFS simulates the flow of passengers. That is, it estimates the number of on-board passengers. First, PFS estimates the number of passengers who appear at stations based on the appearance rate that is calculated from

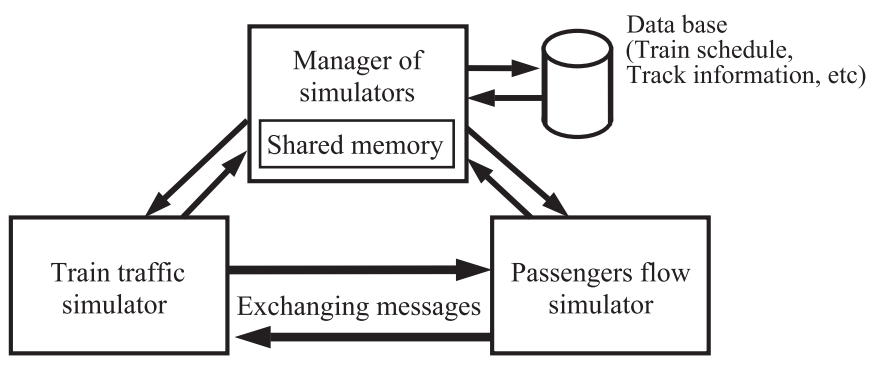

Fig. 3 Structure of the simulator 


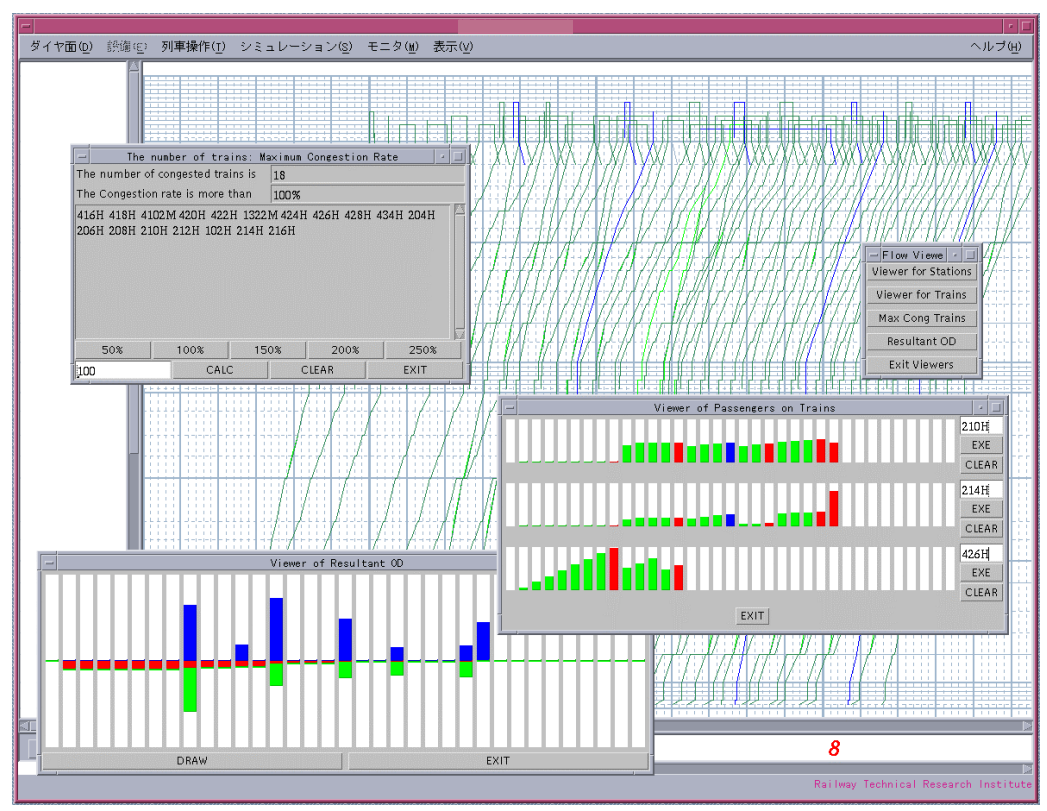

Fig. 4 Display windows of our simulator

the given Origin-Destination (OD) data. When a train arrives at a station, PFS estimates how many passengers get on the train at the station based on the passengers' behavior model presented in the previous section. Moreover, if the train passes the passengers' destination station, the number of the passengers is not added to the number of on-board passengers. The capacity of trains is also taken into account. In other words, the number of on-board passengers does not exceed the capacity.

In order to represent the interaction between the flow of passengers and the delay of trains, we need a model that represents the augmentation of dwell time based on the number of passengers. Hence, we analyze experimental data obtained at the Railway Technical Research Institute. Then, we have the following formula.

(Necessary dwell time)

$=c+p$ (the number of passengers who get on for a door)

$+q$ (the number of passengers who get off for a door)

$+r$ (the number of the current on-board passengers)

.................(6)

where $c, p, q$ and $r$ are parameters obtained from the experimental data.

We can say that the interaction is the relation between the delay of trains caused by congestion of passengers and the congestion caused by the delay. In order to get the resultant dwell time, PFS compares the necessary time calculated by above formula with the current resultant dwell time received from TTS. PFS adopts the larger value as the resultant dwell time and send the value to TTS. If the necessary time is larger than the current resultant dwell time, the corresponding train departure is delayed on TTS. This means that our simulator represents the delay of train departure caused by the increase of passengers. While passengers keep appearing at a station, the delay of train departure can cause augmentation of the number of passengers at the station. This represents the congestion caused by the delay.
Figure 4 shows an overview of our simulator. The background window shows a diagram of the original train traffic schedule together with the simulation results. The front windows show the congestion rates, the number of on-board passengers and the number of passengers waiting at each station.

\section{Case study}

We will show how our simulation method works by the example presented in Fig. 1. The example seems so simple but it is useful to clarify inherent problems. Evaluating Plan 1 (shown in Fig. 1 (a)) and Plan 2 (Fig. 1 (b)) from the viewpoint of passengers, we will show that our method can be useful to support the work of train dispatchers.

\subsection{Case study model}

Table 1 shows the appearance rate of passengers at each station transformed from imaginary OD data. In our case study, the number of passengers is just for one door

Table 1 Appearance rates of passengers

\begin{tabular}{cccccc}
\hline Station No. & St. 0 & St. 1 & St. 2 & St. 3 & St. 4 \\
\hline $\begin{array}{c}\text { Appearance rate } \\
\text { [person/sec] }\end{array}$ & 0.0 & 0.1 & 0.3 & 0.1 & 0.1 \\
$\begin{array}{c}\text { Appearance duration } \\
{[\mathrm{sec}]}\end{array}$ & 0 & 510 & 270 & 450 & 450 \\
\hline
\end{tabular}

\section{Table 2 Total traveling times}

\begin{tabular}{cc}
\hline Plan No. & Total traveling time \\
\hline Plan 1 & $257702[$ person sec] \\
Plan 2 & $252325[$ person sec] \\
\hline
\end{tabular}


of each train. So the capacity of each train is assumed to be 36 persons, which is a quarter of the capacity of a Japanese traditional vehicle with four doors on one side. The appearance duration in Table 1 denotes the time during which passengers appear at stations. For example, the number of passengers who appear at Station 1 is obtained as $0.1 \times 510=51$. Then, the total number of passengers who appear at all stations is 222 . We set the time interval to start just after the departure of Local 0 at each station.

The original schedule drawn in Fig. 1 is based on the values specified as follows. The dwell time of each train at each station is 30 seconds. The running time of Express between any consecutive two stations is 240 seconds, and the running time of other trains is 300 seconds. The initial delay of Local 1 at Station 1 is 120 seconds. The destination station of all passengers is Station 5 . All passengers who appear at each station can reach Station 5 because passengers who wait for the last train Local 2 are forced to get on the train.

\subsection{Evaluation results}

In this section, we show the evaluation results of Plans 1 and 2, and discuss which is a better rescheduling plan from the viewpoint of passengers. This discussion shows that we can determine the better plan quantitatively if we settle criteria.

\subsubsection{The total traveling time}

Table 2 shows the values of the total traveling time that is the sum of all traveling times (including waiting time) of all passengers. Since the value of Plan 2 is smaller than that of Plan 1, we can determine that Plan 2 is a better plan from the viewpoint of the total traveling time. The difference between the values is, however, only two percent. In order to determine the better plan more certainly, we should adopt other criteria such as congestion.

\subsubsection{Congestion rates}

Figure 5 shows the congestion rates of Local 1, Local 2 and Express in case Plan 1 is adopted. The horizontal axis indicates the consecutive two stations as the location of a train. The vertical axis shows the congestion rate of each train. Now, we focus on the congestion rates of Local 1 between Stations 3 and 4. This value is smaller than that between Stations 2 and 3 by approximately $30 \%$. This means that a certain number of passengers transfer from Local 1 to the following trains at Station 3. Consequently, the number of passengers of Local 1 between Stations 3 and 4 decreases, and the number of passengers of Express between Stations 3 and 4 increases.

Figure 6 depicts the congestion rates when Plan 2 is adopted. The congestion rate of Express is lower than that of Local 1 or Local 2, between Stations 2 and 5. In Fig. 5, however, the congestion rate of Express is almost the same with that of Local 2, between Stations 3 and 4 . Since we consider that the congestion of each train should be kept almost the same, the difference of congestion rates at the same section should be small. In order to determine a better plan from the viewpoint of passengers, we

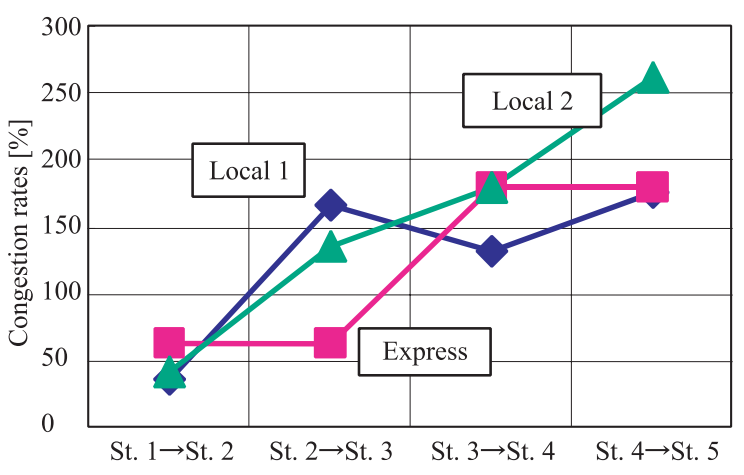

Fig. 5 Congestion rates of Plan 1

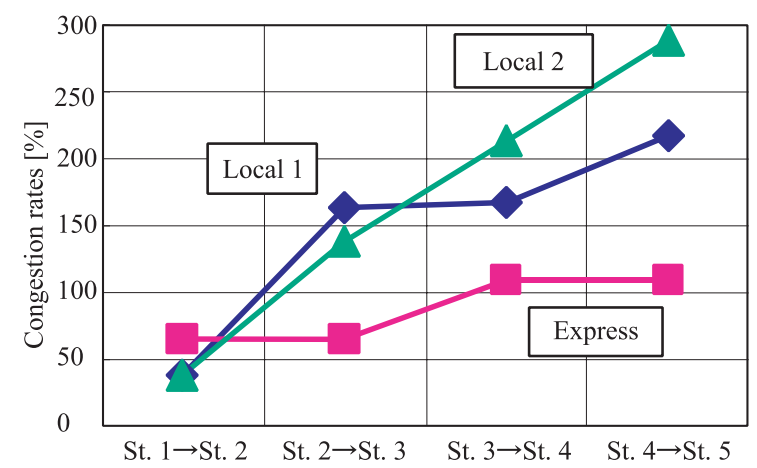

Fig. 6 Congestion rates of Plan 2

introduce a criterion to measure how congestion is averaged among the trains. In the next subsection, we define a deviation of congestion rates.

In Fig. 6, the congestion rate of Local 2 is extremely high between Stations 4 and 5 . The reason is that the train carries all passengers that have waited for the train at all the stations. This is based on the assumption that passengers know Local 2 is the last train bound for Station 5. This assumption keeps same the number of passengers who can reach their destination between the rescheduling plans. Therefore, we can compare the plans with the total number of passengers unchanged.

\subsubsection{The deviation of congestion rates}

Let $c_{i j}$ be the congestion rate of train $j\left(j=1,2, \ldots, N_{i}\right)$ between Stations $i$ and $i+1$ and $m_{i}$ be the average congestion rate between Station $i$ and Station $i+1$. Then, we have the average as

$$
m_{i}=\sum_{j=1}^{N_{i}} c_{i j} / N_{i}
$$

Now, we can denote

$$
a_{i}=\sqrt{\sum_{j=1}^{N_{i}}\left(c_{i j}-m_{i}\right)^{2} / N_{i}}
$$

as a deviation of congestion rates between Stations $i$ and $i+1$. We regard that the congestion is averaged, if the deviation is within a certain limit.

Figure 7 shows the deviations of congestion rates for Plans 1 and 2. Although the values between Stations 1 


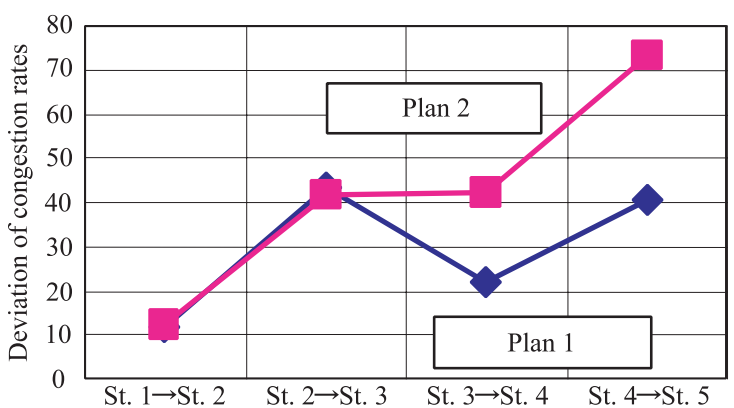

Fig. 7 Deviation of congestion rates

and 3 are almost the same, the values of Plan 1 are smaller than those of Plan 2 between Stations 3 and 5 . Then, we can determine that Plan 1 is better than Plan 2 based on the criterion.

\section{Conclusion}

We have introduced a simulation method to estimate the number of on-board passengers for given traffic rescheduling plans. The method is a combination of train traffic simulator and passengers flow simulator, which can represent the dynamic interaction between train traffic and passengers flow. We can get various kinds of indices useful for evaluation of rescheduling plans based on the number of passengers gained by our method.

Our future work to put the estimation method to practical use is how to obtain actual OD data in detail. We believe that the advance of information technology such as automatic ticket inspection and train operation control systems will realize real time provision of the OD data.

\section{References}

1) Kobayashi, R., Ieda, H., Shibasaki, R. and Terabe, S.: "Evaluation of railway schedule in emergency in terms of "dis"utility of users (in Japanese)," J-RAIL 2000, Kawasaki, Japan., pp.379-382, 2000.

2) Ieda, H. and Nagai, K.: "An Evaluation of Train Scheduling on Commuter Railway with the Analysis of Passengers Behavior of Avoiding Congestion (in Japanese)," J-RAIL'94, Kawasaki, Japan., pp. 368-371, 1994.

3) Ono, K., Kotani, M. and Sakaguchi, T.: "Analysis of Route Choice for Commuter Trips (in Japanese)," RTRI REPORT, Vol.2, No.12, pp. 22-27., 1988.

4) Aoki, T., Ooto H., Tsuzuki, T.: "Reduction of train dwell time by smooth movement of passengers (in Japanese)," Raiway Research Review, No.12, pp. 12-15, 1997.

5) Ben-Akiva, M.E. and Lerman, S.R.: "Discrete Choice Analysis," MIT Press, Massachusetts., 1985.

6) Ono, K., Nozue, N., Kotani, M. and Abe, Y.: "A System for Simulating Train Transportation and an Analysis of Congestion," Quarterly Report of RTRI, Vol.40, No.4, pp. 219-222, 1999.

7) Hirai, C. and Tomii, N.: "A Train Traffic Control Simulation with Passenger's Flowing Model," International Conference on Developments in Mass Transit Systems, IEE (The Institution of Electrical Engineers), London., pp. 74-79, 1998.

8) Sakaguchi, T. and Tomii, N.: "A Train Traffic Model based on Coloured Petri Nets and its Application to a Train Schedule Planning System," Computers in Railways V, Berlin, Germany., Vol.1, pp. 457-466, 1996.

9) Tomii, N. and Sakaguchi, T.: "A Universal Train Traffic Simulation Model and its Applications to Train Scheduling Systems," Europe Multi Simulation Symposium, Istanbul, Turkey., pp. 633-639, 1997. 